\title{
0 Modelo SWAT como Ferramenta para a Gestão de Recursos Hídricos: Um exemplo aplicado no rio Apeú, Castanhal/PA
}

Joyse Tatiane Souza dos Santos Hildo Giuseppe Garcia Caldas Nunes Ana Karla dos Santos Pontes Adriano Marlisom Leão de Sousa
Submetido em: 20/02/18 Revisado em: 15/03/18 Aprovado em: 19/04/18

\section{RESUMO}

O gerenciamento dos recursos hídricos tem impulsionado pesquisas, que buscam alternativas de conhecimento dos processos ocorridos em bacias hidrográficas. Diante disso, este estudo teve como objetivo aplicar o modelo SWAT (Soil \& Water Assessment Tool) como ferramenta complementar para o gerenciamento de recursos hídricos. O modelo foi utilizado na bacia do Rio Apeú, de 2009 a 2017, para a obtenção de informaçōes sobre a dinâmica do ciclo hidrológico, a partir das variáveis climáticas, tipo e uso do solo, estas informaçōes podem auxiliar no planejamento da gestão dos recursos hídricos. Durante a calibraçáo do modelo, foi obtida uma boa eficiência (Nash-Sulticliffe) com um valor de 0,89 , que foi alcançada pela correlação $\left(R^{2}=0,99\right)$ entre os valores simulados e observados e pelos baixos valores de erro durante a simulação da vazão. Estes resultados mostram que o modelo pode auxiliar na geração de informaçôes em áreas que não possuem monitoramento e assim obter um melhor planejamento dos recursos hídricos para as bacias.

Palavras-chave: Modelagem; Hidrologia; Geoprocessamento e Vazão

\section{ABSTRACT}

The water resources management has driven researches, which search for alternatives to the understanding about processes occurring in river basins. Therefore, this study aimed to apply the SWAT model (Soil \& Water Assessment Tool) as complementary tool for water resources management. The model was used in Apeú River Basin, from 2009 to 2017 to obtain information on the hydrological cycle dynamics based on climatic variables, type and land use. This kind of information can help in the planning of water resources management. During the model calibration, it obtained good efficiency (Nash-Sulticliffe) with a value of 0.89 which was achieved by the correlation $\left(\mathrm{R}^{2}=0.99\right)$ between the simulated and observed values and by the low error values during the flow simulation. These results showed that the model can help in the generation of information for areas without monitoring, and consequently it can help the best planning of water resources for basins.

Keywords: Modeling; Hydrology; Geoprocessing and Flow

importante ferramenta de análise e representação simplificada do ciclo hidrológico (FAN; RAMOS; COLLISCHONN, 2015; ALMEIDA; SERRA, 2016).

Dentro desse contexto, pesquisas demonstram modelos hidrológicos auxiliando em estudos ambientais, o qual tem ajudado a entender os impactos das mudanças no uso da terra e projeçóes de alteraçóes futuras no ecossistema, como o caso dos Modelos Hidrológicos de Grandes Bacias (MGB-IPH) (PONestudos no âmbito da modelagem hidrológica, a qual, aplicada ao gerenciamento hídrico, torna-se uma 
TES et al., 2015), o Stanford, o Soil Conservation Service (SCS) (CORREIA et al., 2015), o Stream flow Syntesis and Reservoir Regulation (SSAR) (AHN et al., 2014), o TOPMODEL (METCALFE et al., 2015), o Système Hydrologique Européen (SHE) (DERAEDT et al., 2014), o Institute of Hydrology Distribuited Model (IDHM) (NIU; KNOULFT, 2016) e o Soil and Water Assessment Tool (SWAT) (ARNOLD et al., 1998).

Neste estudo utilizou-se o modelo hidrológico SWAT, devido sua flexibilidade, e por ser uma excelente ferramenta para o fornecimento de subsídios aos órgãos de gestão territorial e de recursos hídricos (GASSMAN; SADEGHI; SRINIVASAN, 2014), além de suas aplicações serem voltadas para o monitoramento de vazóes, projeçóes de enchentes, qualidade da água, processos erosivos, entre outros. Ele foi originado de diversas correçóes e aperfeiçoamento do simulador de recursos hídricos em bacias rurais (SWRRB - Simulator for Water Resources in Rural Basins), desenvolvido pela USDA nos anos 80 , com intuito de gerir os impactos na água e o movimento de sedimentos em bacia rurais sem monitoramento, nos Estados Unidos (ANDRADE, 2013).

Desde a sua criação a ferramenta tem passado por inspeçóes contínuas e expansão da sua capacidade, entre elas são: SWAT 94.2 (incorporação das unidades de respostas múltiplas - URHs); SWAT 96.2 (autofertilização e irrigação); SWAT 98.1 (aprimoramento da qualidade do fluxo de água); SWAT 99.2 (melhoria nas rotinas do ciclo de nutrientes); SWAT 2000 (transporte de bactérias); entre outros. Além dessas mudanças, também foram desenvolvidas interfaces do modelo com os programas Windows (visual basic), GRASS, ArcGis e atualmente o QGis, bem como extensivo processo de validação (NEISTCH et al., 2011).

O modelo é reconhecido internacionalmente como uma robusta e interdisciplinar ferramenta de modelagem hidrológica, o que é evidenciado pelas conferências internacionais sobre o SWAT (Brasil/2014; Itália/2015; China/2016; Malásia/2017), e publicaçôes relacionadas ao modelo, apresentadas em diversos encontros e revistas científicas (WANG et al., 2010; BRESSIANI et al., 2015; DILE et al., 2016). $\mathrm{O}$ modelo tem sido amplamente difundido graças aos avanços na tecnologia, o qual tem comprovado ser uma ferramenta flexível que pode ser aplicada para simular diversos problemas em uma bacia hidrográfica (KUWAJIMA et al., 2011).

Como por exemplo, no estudo de Wang et al. (2010) que examinaram os efeitos interativos do uso e do tipo do solo, sobre a vazão de água e os teores de sedimentos, na bacia hidrográfica de Cowhouse Creek, localizada no norte do Texas, com o objetivo de desenvolver melhores práticas de manejo do solo, auxiliando na saúde da população, bem como, na sustentabilidade da bacia, relacionados aos efeitos do uso da terra.

Já no Brasil, por apresentar proporçóes continentais, permite uma grande variabilidade climática, entre as diferentes regióes, assim como a ocorrência de diferentes biomas, logo há uma maior necessidade de ferramentas no auxílio à tomada de decisóes, no que se refere ao manejo dos recursos naturais (BRESSIANI et al., 2015). Recentemente, Blainski; Acosta; Nogueira (2017) mostraram a eficácia do modelo SWAT na representação do fluxo hidrossedimentológico em uma bacia hidrográfica (rio Camboriú), localizada em Santa Catarina, a partir da calibraçáo e validação do modelo, com o intuito de comprovar a eficiência do SWAT na simulação das curvas de permanência das diversas variáveis estudadas, demonstrando o uso do modelo como uma ferramenta importante na gestão de bacias hidrográficas.

Tendo em vista a importância de obtenção de informaçóes sobre a dinâmica de uma bacia, no seu gerenciamento e monitoramento, optou-se por aplicar a modelagem hidrológica, na bacia do rio Apeú, que apresenta um constante crescimento populacional, provocando mudanças no uso e cobertura do solo. Ainda ao se tratar de uma área com diversas nascentes contribuintes ao rio Guamá, sendo necessário um melhor planejamento e gerenciamento dos recursos hídricos.

Assim, este artigo tem como objetivo propor a aplicabilidade do modelo SWAT como ferramenta complementar no gerenciamento de recursos hídricos, usando como exemplo a bacia hidrográfica do Rio Apeú, mostrando que é possível a utilização do modelo na obtenção de informações sobre a dinâmica de uma bacia, e com isso, representando o regime hidrológico, a partir das variáveis climáticas, tipo e uso do solo. 


\section{MATERIAIS E MÉTODOS}

\section{Área de Estudo}

A bacia hidrográfica do rio Apeú, faz parte do conjunto de sub-bacias do Rio Guamá, localizada no nordeste do estado do Pará, na mesorregião metropolitana de Belém. A Sub-bacia se estende entre as coordenadas, $1^{\circ} 13^{\prime} 10^{\prime \prime}$ e 1'27'37" de latitude Sul e 4804'42” e 47053’30" de longitude Oeste, com área de aproximadamente $307 \mathrm{~km} 2$, pertences aos Municípios de Castanhal, Santa Izabel do Pará e Inhangapi (Figura 1).

O Clima regional é do tipo Ami, de acordo com a classificação de Koppen, as temperaturas máximas e mínimas são $35^{\circ}$ e $18^{\circ} \mathrm{C}$, respectivamente, com temperatura média anual de $26^{\circ} \mathrm{C}$. A umidade relativa média e chuva anual são de $80 \%$ e $2.571,6 \mathrm{~mm}$, respectivamente (TENÓRIO et al., 1999; SOUZA, 2017).

\section{Obtençáo dos dados}

O tipo do solo foi determinado a partir da base de dados do Instituto Brasileiro de Geografia e Estatística (IBGE), na escala de 1:250.000, aonde foi encontrada duas classes de solos, distribuídos da seguinte forma: 92\% Latossolo Amarelo e 8\% Gleissolo Háplico. Com relaçáo ao uso do solo da bacia em estudo, o mapa foi elaborado com base no projeto TerraClass, desenvolvido pelo Instituto Nacional de Pesquisas Espaciais (INPE), a partir de imagens de Satélites LANDSAT-5, com resolução espacial de $30 \mathrm{~m}$, em que neste estudo utilizou a órbita/ponto 223/061, gerando sete classes (agricultura anual, pasto, área urbana, floresta, vegetação secundária, mineração e hidrologia), as quais foram reclassificadas com base no banco de dados do modelo Hidrológico SWAT em: As Áreas Antrópicas Agrícolas recobrem 41\% da área. As Áreas Urbanas recobrem 12\%. As Pastagens recobrem

Figura 1 - Localização do rio Apeú, Pará, Brasil

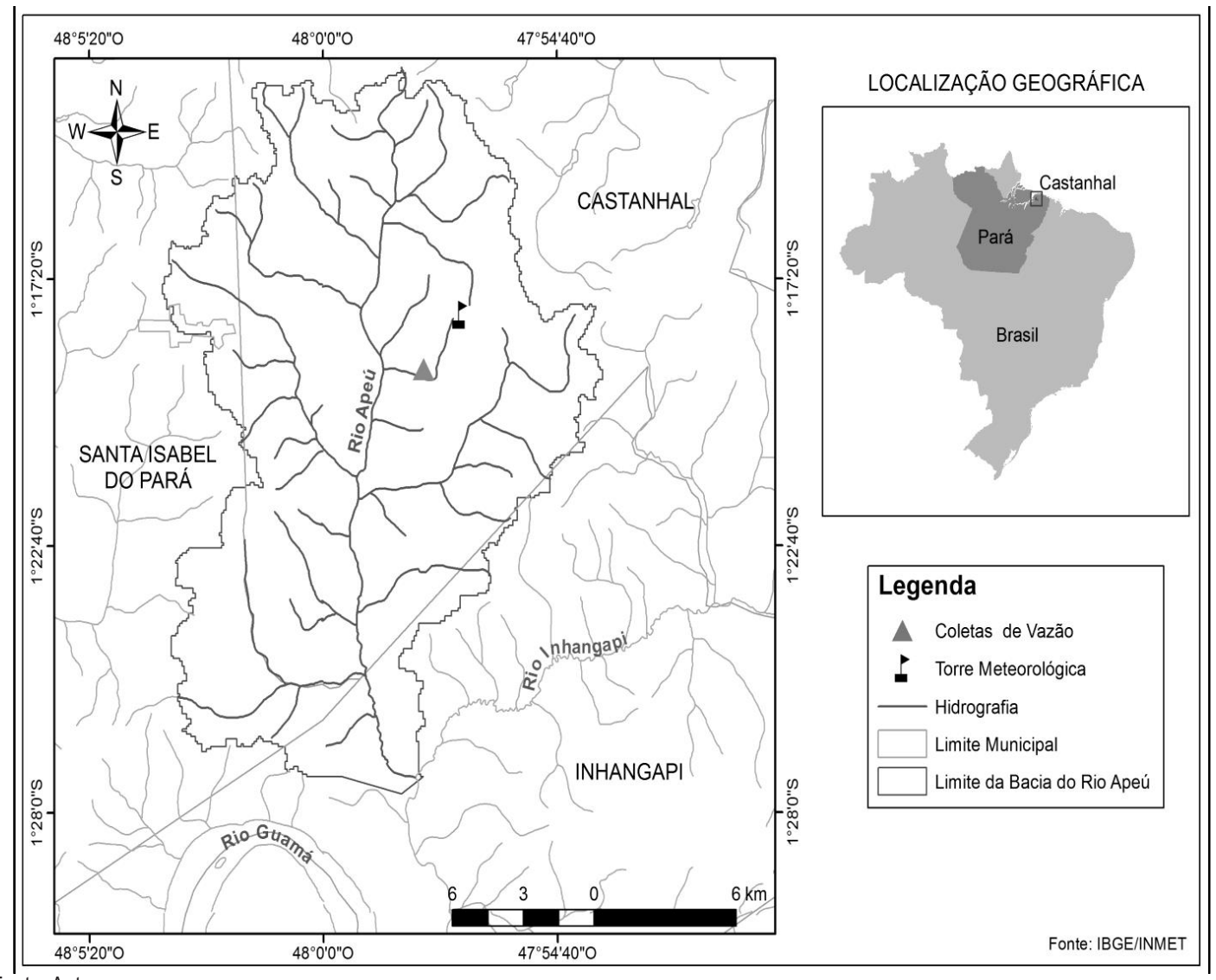


11\%, A Floresta Mista recobre 35\%, A Mineração e os corpos hídricos ocupam menos de $1 \%$ da área.

O MNT da sub-bacia foi obtido gratuitamente do site da Empresa Brasileira de Pesquisa Agropecuária (EMBRAPA), a partir de imagens SRTM, com resolução espacial de $90 \mathrm{~m}$. Posteriormente foi transformada em imagens raster, de resoluçâo espacial de $30 \mathrm{~m}$, na escala de 1:250.000, assim como as informaçóes de tipo e uso do solo envolvidos neste estudo, pois elas devem possuir a mesma resolução espacial e mesma escala para serem adicionadas no modelo.

Os dados climáticos utilizados foram de temperatura máxima e mínima (Tmax e Tmin), velocidade e direção do vento (VV e DV), umidade relativa (UR), radiação solar (RS), ponto de orvalho (PO) e precipitação (PRP), no período de 2009 a 2017, obtidos de uma torre meteorológica, do Instituto nacional de meteorologia (INMET) localizado no município de Castanhal/PA.

As mediçôes de vazóes foram realizadas através do método convencional, com a utilizaçáo do molinete hidrométrico, para a determinação da área e da velocidade média do fluxo que passa pela seçáo transversal do ponto monitorado (MALDONADO; WENDLAND; PORTO, 2015). De acordo com a medição a vau (Medição realizada por um observador que atravessa o curso de água a pé medindo profundidades e velocidades, indicados para rios de pequena largura e pouca profundidade (CARVALHO, 2008)), dividiu-se em três seçóes $(0,5 \mathrm{~m}, 1 \mathrm{~m}$ e $1,5 \mathrm{~m})$, ao longo do comprimento total $(2,20 \mathrm{~m})$ da seção transversal do tributário do rio Apeú (localizado na sub-bacia 5), em nível d'água de $0,14,0,14$ e $0,11 \mathrm{~m}$ (60\% de profundidade), durante 30 dias (de novembro a dezembro), conforme resolução da ISO 748 (ISO, 2007).

O cálculo do número de rotação por segundo no molinete, se dar pela divisão entre número de rotaçôes registradas e o intervalo de tempo de medição da velocidade. Posteriormente este valor é convertido em velocidade através da equaçáo do molinete, a qual é calibrada para cada molinete de forma individual, neste estudo foi usado o molinete JCTM, de modelo MLN-7 (Equação 1):

$$
V=a x N+b
$$

Onde,
$\mathrm{V}=$ Velocidade;

$\mathrm{a}=$ Passo da hélice;

$\mathrm{b}$ = Inércia da hélice;

$\mathrm{N}=$ Número de rotação por segundo.

A vazão foi calculada pelo método da meia seção, que consiste no cálculo das vazóes parciais, por meio da multiplicação da velocidade média na vertical pelo produto da profundidade média na vertical e pela soma das semidistâncias às verticais adjacentes (vazão parcial determinada para cada região de influência de uma determinada vertical) (ANA, 2016) (Equação 2) e a vazão total (Equação 3):

$$
q_{i}=V_{i} h_{i}\left(\frac{d_{i+1}-d_{i-1}}{2}\right)
$$

Onde,

$q_{1}=$ Vazão na seção i $(\mathrm{m} 3 / \mathrm{s})$;

$v_{1}=$ Velocidade média na vertical i $(\mathrm{m} / \mathrm{s})$;

$h_{1}=$ Profundidade da vertical i $(\mathrm{m})$;

$d_{1+1}=$ Distância a partir do PI até a vertical posterior $(\mathrm{m})$;

$d_{1-1}=$ Distância a partir do PI até a vertical anterior (m).

$$
=\sum_{i}^{n} q_{i}
$$

\section{Processamento dos dados: Modelo Swat}

O SWAT avalia o efeito do uso do solo no escoamento, no transporte de sedimento e na qualidade da água em bacias hidrográficas (ARNOLD et al; 2012; BRIGHENTI; BONUMÁ; CHAFFE, 2015). O seu funcionamento ocorre basicamente pelas entradas de dados na interface SIG (Sistema de Informação Geográfica), organizados em dados geocartográficos e tabulares, os quais correspondem aos mapas de uso e cobertura da terra, mapas pedológicos, hidrografia e modelo numérico de terreno (MNT) e dados climáticos (velocidade do vento, precipitação, temperatura, radiação solar, umidade relativa do ar) respectivamente. Nesse estudo foi utilizado o SWAT, na versão 2012 (Figura 2).

A simulação hidrológica realizada pelo modelo é dividida em duas fases. A primeira consiste na fase terrestre do ciclo hidrológico, a qual controla a quantidade de água e sedimentos para o canal principal de cada sub-bacia. Enquanto que a segunda é a fase de propagação de canal (vazão), representando o balanço hídrico, conforme a equação 4 (NEITSCH et al., 2009). O balanço hídrico é a força motriz que impulsiona a execução de todos 
Figura 2 - Processo de Funcionamento do Modelo SWAT

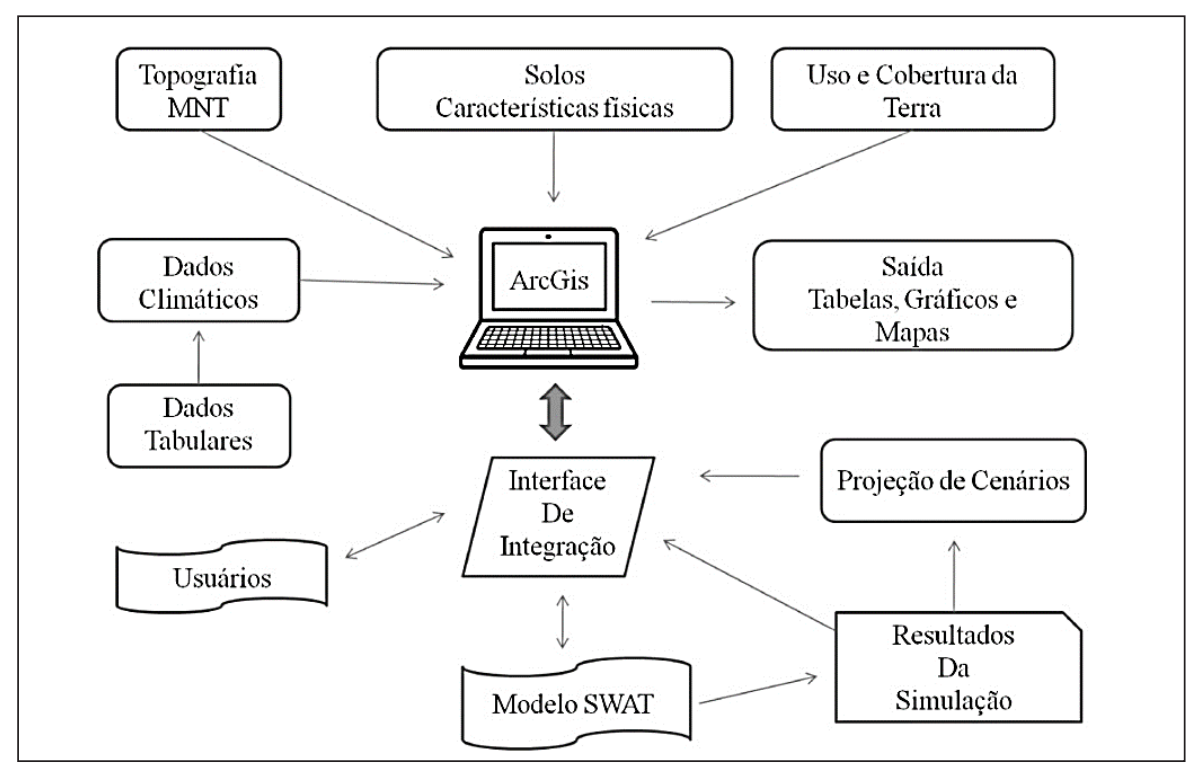

Fonte: adaptado de SOUSA, 2010

os processos no modelo SWAT (FERRIGO et al., 2014). Ele incorpora oito componentes principais para prover suas simulaçóes (ARNOLD, 1998), são eles: a - Hidrologia (escoamento superficial, percolação, fluxo lateral, fluxo subterrâneo, evapotranspiração, neve e o fluxo da rede de drenagem). Incluindo ainda a análise em reservatórios; b - Aporte sedimentos; c - Clima; $\mathrm{d}$ - Crescimento vegetal; e - Nutrientes (investiga os compostos minerais e orgânicos do nitrogênio e fósforo) e; f - Pesticidas e bactérias.

$$
S W_{t}=S W_{O}+\sum_{i=1}^{t}\left(R_{i}-Q_{i}-E t_{i}-P_{i}-Q R_{i}\right)
$$

Onde:

$S W_{t}=$ É a quantidade final de água no solo $(\mathrm{mm})$;

$S W_{o}=$ É a quantidade inicial de água no solo $(\mathrm{mm})$;

$t=$ É o tempo (dias);

$R_{i}=$ É a precipitação (PRP) $(\mathrm{mm})$;

$Q_{i}=$ É o escoamento superficial $(\mathrm{mm})$;

$E T_{i}=$ É a evapotranspiração $(\mathrm{mm})$;

$P_{i}=$ É a percolação $(\mathrm{mm}) ; \mathrm{e}$,

$\mathrm{Q} R_{i}=$ É o fluxo de retorno (é o escoamento de água no interfluxo, sobre a superfície do terreno, após emergir da superfície, ocorrida através da ascensão capilar) (mm).
O modelo SWAT divide a bacia em sub-bacias com base no relevo, solos e uso da terra, preservando assim os parâmetros espacialmente distribuídos da bacia inteira e características homogêneas dentro da bacia. Essas sub-bacias são divididas em URHs, que representam combinaçóes únicas de uso da terra, tipo do solo e classe de declividade. As URHs não interagem entre si. As respostas de cada URH (escoamento, sedimentos e nutrientes) são calculadas separadamente e entáo somadas para encontrar-se a carga total da sub-bacia (PERAZZOLI; PINHEIRO; KAUFMANN, 2013).

\section{SWAT-CUP}

O programa integra cinco procedimentos de calibração e análise de incertezas semiautomáticas: SUFI2, PSO, GLUE, ParaSol e MCMC, para a interface do modelo SWAT (BRIGHENTI; BONUMÁ; CHAFFE, 2015), para este estudo foi aplicado o método SUFI2 (Sequential Uncertainty Fitting), o qual utiliza o método do hipercubo latino para definição dos parâmetros, a calibração com o SUFI-2 é realizada através de sucessivas iteraçóes, com o mesmo número de simulaçóes cada. A cada iteração o intervalo de valores dos parâmetros calibrados é reduzido, sempre 
Tabela 1- Equaçóes para o cálculo do tempo de concentração (tc)

\begin{tabular}{c|c|c}
\hline Parâmetro & Descrição & Faixa de variação \\
\hline CN2 & Coeficiente de rugosidade do canal principal $(\mathrm{m}-1 / 3 \mathrm{~s})$. & 35 a 98 \\
\hline ALPHA_BF & Fator do fluxo de base (dia). & 0 a 1 \\
\hline GW_DELAY & Intervalo de tempo para recarga do aquífero (dias) & 0 a 500 \\
\hline REVAPMN & Percolação para o aquífero profundo & 0 a 500 \\
\hline DEEPST & Profundidade inicial de água no aquífero profundo $\left(\mathrm{mm} \mathrm{H}_{2} \mathrm{O}\right)$ & 0 a 50000 \\
\hline GWQMN & Nível limite de água no aquífero livre para ocorrer o fluxo de retorno & 0 a 5000 \\
\hline SOL_AWC & Armazenamento de água no solo (mmH2O mmsolo-1) & 0 a 1 \\
\hline ESCO & Coeficiente de compensação de evaporação de água no solo & 0 a 1 \\
\hline GW_REVAP & Coeficiente de ascensáo de água à zona de saturaçáo & 0.02 a 0.2 \\
\hline RCHRG_DP & Coeficiente de percolação da água para o aquífero profundo $(\mathrm{mm})$ & 0 a 1 \\
\hline SURLAG & Coeficiente de retardamento do escoamento superficial direto & 0.05 a 24 \\
\hline CH_N2 & Coeficiente de Manning para o canal principal & -0.01 a 0.3 \\
\hline SLSOIL & Comprimento da declividade para o escoamento subsuperficial $(\mathrm{m})$ & 0 a 150 \\
\hline LAT_TTIME & Escoamento lateral (dias) & 0 a 180 \\
\hline CANMX & Água interceptada (mm) & 0 a 100 \\
\hline
\end{tabular}

centralizado no conjunto que obteve o melhor valor para a função objetivo (FRANCO; OLIVEIRA; BONUMÁ, 2017).

No SUFI-2 as incertezas das estimativas são comunicadas através de dois indicadores estatísticos: o fator-p e o fator-r. O fator-p indica a porcentagem de dados observados que é englobada pelo resultado de simulaçóes ao final da iteração. $O$ fator-p varia de 0 a 1 , onde 1 corresponde a $100 \%$ dos dados observados. $\mathrm{O}$ fator-r indica a espessura do resultado de simulações, isto é, a incerteza da iteração. Um modelo está calibrado quando se atinge um equilíbrio entre ambos os fatores, englobando parcela razoável dos dados observados, com um nível de incertezas aceitável (FRANCO; OLIVEIRA; BONUMÁ, 2017). Valores acima de 0,70 para o fator-p e abaixo de 1,50 para o fator-r indicam uma calibração aceitável de vazão (ABBASPOUR, 2015).

Quinze parâmetros foram escolhidos para calibração, para determinar quais variáveis são mais influentes e importantes para o escoamento superficial. Foram efetuadas 2 iteraçôes, padronizadas com 500 simulações cada (ABBASPOUR, 2015), até a obtenção de um intervalo (valores mínimos e máxi- mos) adequado às condiçóes da bacia. Os parâmetros do modelo obtidos na calibração, a descrição e sua faixa de variação admitida no programa Swat-cup encontram-se na Tabela 1.

A simulaçáo do modelo foi no período de 2009 a 2017. Para a etapa de calibração, foram comparados os valores de vazáo simulados com os valores de vazóes observados, para o período de três meses (de outubro a dezembro de 2017). Após a calibração foi realizada uma análise de sensibilidade global. O melhor desempenho do modelo é obtido a partir dos valores observados e simulados, calculados com base nos seguintes métodos estatísticos: o coeficiente de eficiência de Nash-Sutcliffe $\left(\mathrm{NS}_{\mathrm{Ef}}\right)$ (equação 5) (varia do $-\infty$ até 1 ), (NASH and SUTCLIFFE, 1970), e o coeficiente de determinação $\mathrm{R}^{2}$ (varia de 0 a 1 ), sendo 1 o indicativo de simulação perfeita de evento simulado para ambos. Moriasi et al., (2007) apresentaram a seguinte classificação para o coeficiente $\mathrm{NS}_{\mathrm{Ef}}$ em simulaçóes diárias: quando, $0,75<\mathrm{NS}_{\mathrm{Ef}}>$ 1,00 o modelo é considerado muito bom; o modelo é considerado bom quando, $0,65<\mathrm{NS}_{\mathrm{Ef}}<0,75$; já entre 0,50 e 0,65 o $\mathrm{NS}_{\mathrm{Ef}}$ é satisfatório; e abaixo de 0,50 é considerado inadequado. 
Foi calculado também o erro do modelo, através da estatística PBIAS (\%) (equação 6), que mede a tendência média dos valores simulados serem maiores ou menores do que os dados observados, sendo o seu valor ideal próximo e/ou igual a 0 . Valores positivos indicam que a simulação subestimou os valores observados, enquanto que valores negativos indicam uma superestimativa (BUENOS et al., 2017). Van Liew et al. (2007) apresentaram a seguinte classificação para este coeficiente, em aplicações do modelo SWAT: quando o $\mid$ PBIAS $\mid<10 \%$, é considerado "muito bom"; ele é considerado "bom", quando o valor está entre, $10 \%<\mid$ PBIAS $\mid<15 \%$; já entre, $15 \%<\mid$ PBIAS $\mid<25 \%$ é considerado "satisfatório"; o modelo está inadequado quando, $\mid$ PBIAS $\mid>25 \%$ (BUENOS et al., 2017).

O modelo foi avaliado, através das equaçóes:

$$
\begin{aligned}
& N S_{E f}=1 \frac{\sum_{1}^{n}\left(Q_{0}-Q_{c}\right)}{\sum_{1}^{n}\left(Q_{0}-\overline{Q_{0}}\right)} \\
& P B I A S=100\left[\frac{\sum_{1}^{n}\left(Q_{0}-Q_{c}\right)}{\sum_{1}^{n} Q_{0}}\right]
\end{aligned}
$$

Onde, $Q o$ é a vazão observada, $\overrightarrow{Q o}$ é a vazão média observada e $Q c$ é a vazão estimada, $\mathrm{m}^{3} \mathrm{~s}^{-1}$.

\section{RESULTADOS E DISCUSSÓES}

Diversas são as respostas que o modelo SWAT pode gerar para quem o utiliza como ferramenta de planejamento relacionado à gestão hídrica. Primeiramente ele é capaz de gerar diferentes unidades organizadas (usos da terra, tipo de solos com propriedades diferentes e declividade), permitindo entender e/ou analisar as diferentes fraçôes de áreas distribuídas por toda a área da bacia. Neste estudo levou-se em consideração a URH dominante de cada sub-bacia, desconsiderando as classes que apresentaram menores representatividades, como resultado o modelo delimitou a bacia do rio Apeú com uma área de aproximadamente $307,0 \mathrm{Km}^{2}$, subdividida em 22 sub-bacias, formando 22 URHs (Figura 3).

O processo de calibração e avaliação de eficiência do modelo, realizada no Swat-cup, identificou um fator-p de valor 0.8 e um fator-r, de 0.9 , mostrando que há um equilíbrio entre os fatores, e, portanto, demonstra uma calibração "aceitável”. Dentre

Figura 3 - Composição das unidades de resposta hidrológica (URH) a partir dos mapas de tipo de solo, cobertura vegetal e topografia

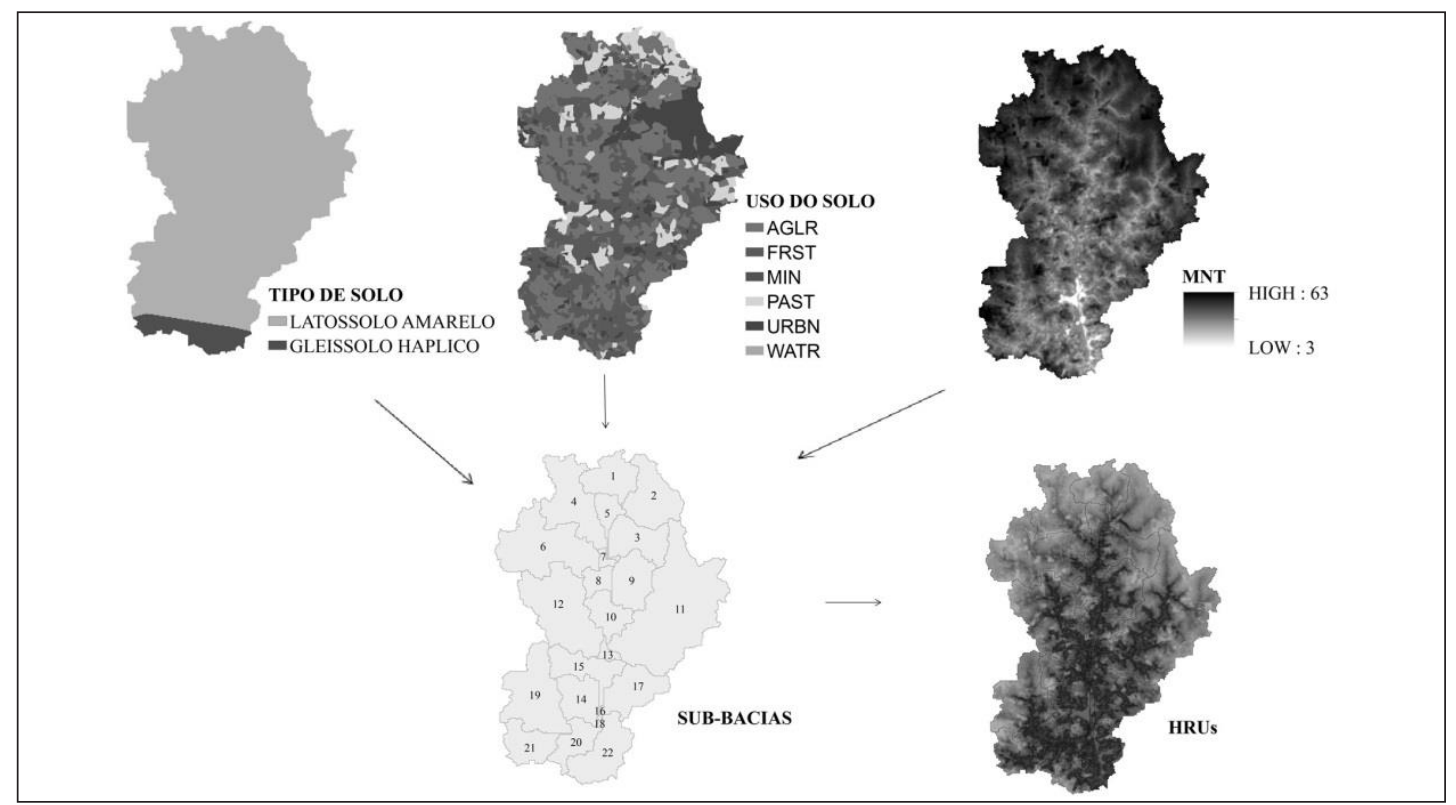

Fonte: Adaptado de EASTON et al., (2008) 
os quinzes parâmetros físicos escolhidos, durante a etapa de calibração, os que apresentaram maior sensibilidade possuem o seu valor de $\mathrm{P}$ próximo à zero, determinando a significância da sensibilidade (ABBASPOUR, 2014). Sendo que, nesse estudo o parâmetro que possui maior influência na modelagem da vazão, é SOL_AWC, que está associado à capacidade de água disponível no solo, ou seja, indicando que existe pouca contribuição de água subterrânea neste perímetro do rio, o que é compatível com as condiçóes pedológicas da sub-bacia (5), predominantemente do tipo latossolo amarelo, caracterizado por solos secos e duros (IBGE 2010). O segundo parâmetro foi o GW_DELAY, associado ao intervalo de tempo para recarga do aquífero, indicando o tempo de atraso das águas subterrâneas, tornando este, um dos parâmetros que tem mais impactos sobre a variável vazão (Figura 4). Este tipo de análises mostra que para se obter um melhor resultado é necessário que os parâmetros sejam analisados e calibrados, conforme a saída desejada do modelo (vazão, sedimento, nutrientes, e etc.) e as características da bacia em estudo.

Figura 4 - Ranking dos parâmetros obtido com a análise de sensibilidade

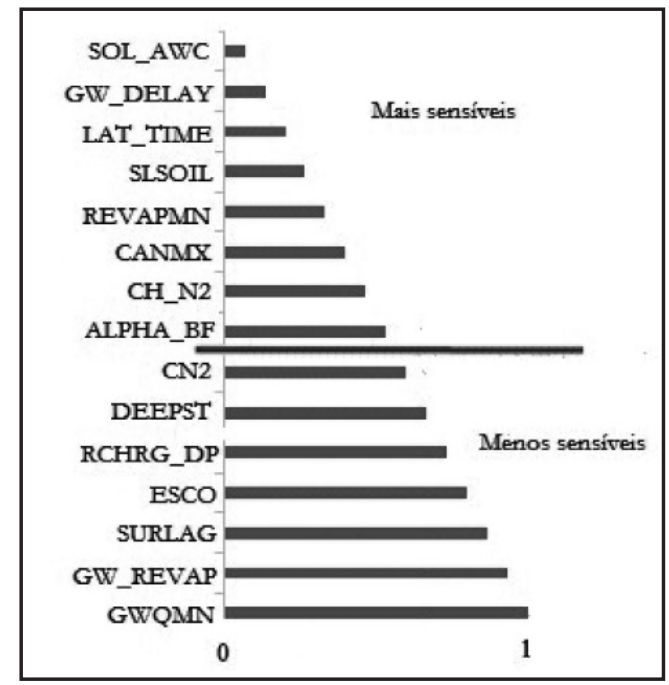

Fonte: Adaptado de Castro (2016)

Durante a calibração, o modelo apresentou uma boa eficiência $\left(\mathrm{NS}_{\mathrm{Ef}}=0,89\right)$ na simulação da vazão, decorrente da alta correlação entre os valores simulados e observados $\left(\mathrm{R}^{2}=0,99\right)$ e dos baixos erros $($ PBIAS $=$
8,8\%) durante as simulaçóes (Figura 5). Este resultado demonstra que o SWAT pode auxiliar na simulação da vazão na bacia do rio Apeú, pois diversos estudos para bacias de mesmo porte apresentaram resultados parecidos. Como no estudo de Silva e Christofaro (2017), que demonstraram valores de $\mathrm{NS}_{\mathrm{Ef}}$ e $\mathrm{R}^{2}$, de 0,53 e 0,62 , respectivamente na calibração do rio Jequitinhonha, no estado de Minas Gerais. E no estudo de Ferreira e Uagoda (2016) que encontraram para os resultados diários, durante a etapa de calibração, os valores de $\mathrm{NS}_{\mathrm{Ef}}$ igual a 0,53 e uma correlação $\left(\mathrm{R}^{2}\right)$ de 0,63 entre os valores de vazão simulada e observada na bacia hidrográfica de Ribeirão do Gama, no Distrito Federal. Assim, os modelos hidrológicos podem ser úteis para uma gestão eficiente de recursos hídricos, principalmente em bacias hidrográficas de pequeno porte ou em regióes onde se tenham conflitos pelo uso da água (BLAINSKI et al.,2017).

Outra resposta do modelo, que pode ser aplicada na gestão dos recursos hídricos, é a simulação do balanço hídrico, que se constitui em uma determinaçáo da variação hídrica local, sem que haja a necessidade de medidas diretas das condiçóes do solo (PEREIRA, 2005; FENNER et al; 2014). Essa informação permite conhecer a disponibilidade hídrica de uma bacia hidrográfica, se há uma deficiência ou excedente hídrico, bem como o armazenamento de água no solo, e ainda permite a estimativa da evapotranspiração local (FENNER et al; 2014). Esse conhecimento colocaria em evidência a importância das áreas de preservação permanente (APP), que a lei de n. 12.695 de 2012 dispóe, destacando a proteção das áreas no entorno dos rios, evitando modificaçóes do uso do solo de forma impactante, que venha influenciar no aumento do escoamento superficial da água e com isso trazer sérios problemas, como, erosão, enchentes, contaminação, e entre outros.

A precipitação anual média (2009 a 2017) para a bacia do rio Apeú foi de $1449,05 \mathrm{~mm}$, deste valor, $583,7 \mathrm{~mm}$ ( $40 \%$ do total de precipitação) retornam por evapotranspiração, ligados aos diversos tipos de uso da terra. Do total de chuva, cerca de $328,78 \mathrm{~mm}$

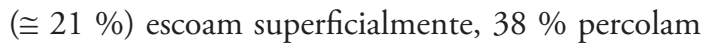
$(529,48 \mathrm{~mm})$ e apenas $1 \%$ revapora $(20,72 \mathrm{~mm})$. Dos $529,48 \mathrm{~mm}$ que percolaram, $1,72 \mathrm{~mm}(\cong 2 \%)$ escoam lateralmente, $\cong 6 \%(26,47 \mathrm{~mm})$ vão para o aquífero profundo e $482,15 \mathrm{~mm}(92 \%)$ retornam a bacia (Figura 6). 
Figura 5 - Comparação entre a vazão simulada (linha) e a vazão observada (circulo), no período de calibração (outubro a dezembro de 2017) do modelo SWAT e a precipitação (coluna) observada no período

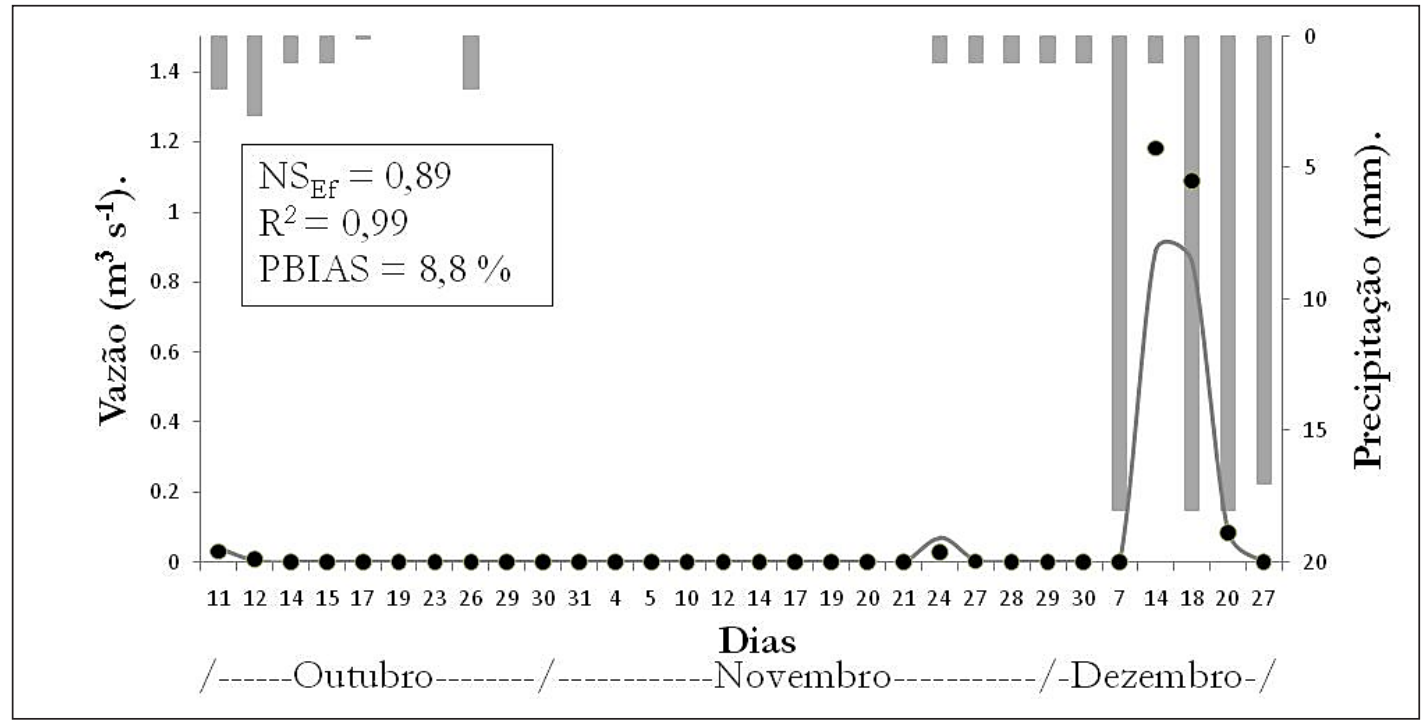

Figura 6 - Ciclo Hidrológico anual simulado pelo modelo SWAT, para a bacia hidrográfica do rio Apeú

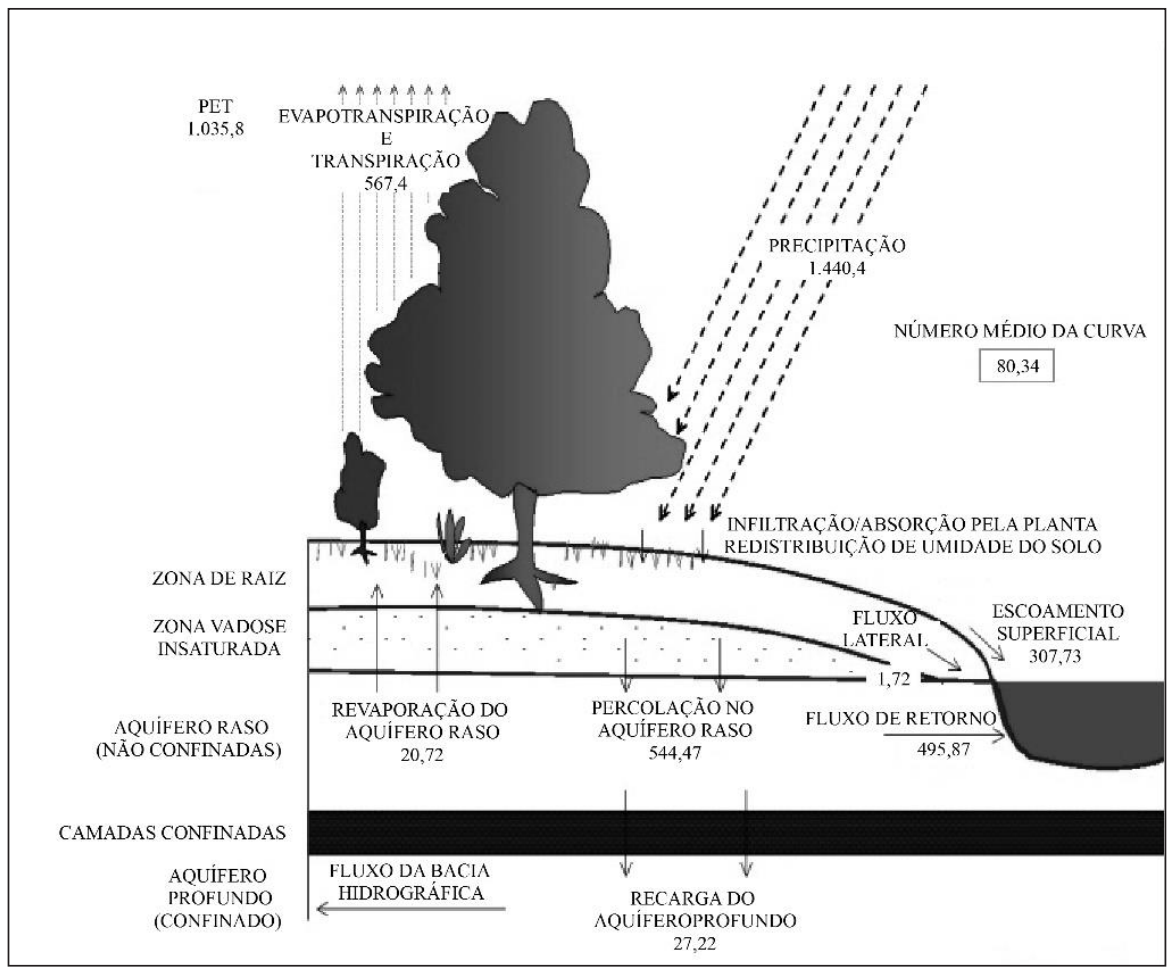


De acordo com o modelo, a bacia do rio Apeú, mostrou que do total de chuva (1449,05 mm), 38\% é percolado $(529,48 \mathrm{~mm}$ ) se convertendo em escoamento e contribuindo com a vazão do rio, com isso, pode-se afirmar que durante esses 9 anos $6 \%$ do volume percolado se destinaram ao aquífero profundo, sendo capaz de colaborar com o aquífero Barreiras.

O SWAT ainda permite, uma análise de cenários que ele simula em função do uso e mudança do solo, demonstrando a influencia dessas alteraçôes sobre o regime hidrológico, com isso é possível com base nos dados de precipitação, visualizar e preencher falhas em vazóes, para um controle de cheias, importante para projetos de hidráulicas (PEREIRA et al, 2016), bem como evitar erosóes, comprometimentos na qualidade da água, etc. Onde percebe-se que as técnicas de modelagem aplicada aos estudos hidrológicos são uma forma prática, rápida e barata de se simular cenários futuros dos recursos naturais, possibilitando a proposição de soluçóes para contornar alguma situação subsequente indesejada (BONUMÁ et al, 2015).

\section{CONCLUSÁO}

O modelo SWAT aplicado a bacia do rio Apeú, mesmo com dados limitados para inserção ao modelo, demonstrou que foi possível delinear a sua área e simular os componentes do balanço hídrico, e em especial a vazão. O uso do modelo SWAT tem se intensificado em estudos de rios brasileiros, em detrimento da quantidade de afluentes existentes sem o monitoramento, devido às dificuldades de acesso a esses rios e pela falta de interesses em aplicar recursos voltados ao seu monitoramento, os quais tem sido muitos evidenciados pelas intensidades de chuvas e prejuízos na região.

Ainda o uso do modelo pode auxiliar no planejamento dos recursos hídricos, uma vez que, o seu uso pode antecipar situaçóes que podem ser prejudiciais á gestáo desses recursos, decorrente dos diversos usos do solo existente na bacia, bem como no gerenciamento da água pelo uso da agricultura, atividade que utiliza bastantes recursos e demanda hídrica.

Contudo, o modelo SWAT também pode ser aplicado em estudos futuros na bacia do rio Apeú, como por exemplo, aos estudos voltados à quantidade do rendimento de água, desde a nascente até seu exutório, com o intuito de entender o quanto as sub-bacias estão contribuindo para o rio principal, e com isso, poder auxiliar no planejamento da quantidade e qualidade da água que chega às comunidades, auxiliando na manutenção dos recursos e melhor uso da água pelos diferentes usos existentes ao longo da bacia. Diante disso, essa pesquisa propóe o quanto o uso do modelo SWAT pode contribuir com o planejamento e nas tomadas de decisão nas bacias.

\section{Referências}

ABBASPOUR, K.C. SWAT-CUP 2012: SWAT Calibration and Uncertainty Programs - A User Manual. Eawag: Swiss Federal Institute of Aquatic Science and Technology. 2014.

ABBASPOUR, K. C. SWAT-CUP: SWAT Calibration and Uncertainty Programs-A User Manual. Eawag: Swiss Federal Institute of Aquatic Science and Technology. 2015.

AGÊNCIA NACIONAL DA ÁGUA. Conjuntura dos recursos hídricos no Brasil: informe 2016. Brasília: ANA, 2016.

AHN, R. J. M; LEE, S; KANG, T. Evaluation of dams and weirs operating for water resource management of the Geum River. Science of the Total Environment, 2014.

ALMEIDA, L.; SERRA, J. C. V. Modelos hidrológicos, tipos e aplicações mais utilizadas. Rev. FAE, Curitiba, v. 20, n. 1, p. 129 - 137, jan. - jun. 2017.

ANDRADE, M. A; MELLO, C. R; BESKOW, S. Simulação hidrológica em uma bacia hidrográfica representativa dos Latossolos na região Alto Rio Grande, MG. Rev. bras. eng. agríc. ambient. vol.17 no.1 Campina Grande, Jan. 2013. 
ARNOLD, J. G. J. R. WILLIAMS, R. SRINIVASAN, KING. K.W. SWAT: Soil and Water Assessment Tool. Temple, TX: USDA-ARS, Grassland, Soil and Water Research Laboratory, 1998.

ARNOLD, J. G.; MORIASI, D. N.; GASSMAN, P.W.; ABBASPOUR, K. C.; WHITE, M. J.; SRINIVASAN, R.; SANTHI, C.; HARMEL, R. D.; VAN GRIENSVEN, A.; VAN LIEW, M. W.; KANNAN, N.; JHA, M. K. SWAT: Model use calibration and validation. Trans. ASABE, v. 55, n. 4, p. 1494- 1508, May 2012.

BLAINSKI, É; ACOSTA, E.; NOGUEIRA, P. C. do P. Calibração e validação do modelo SWAT para simulação hidrológica em uma bacia hidrográfica do litoral norte catarinense. Rev. Ambient. Água, vol. 12 n. 2 Taubaté, Mar. -Apr. 2017.

BRESSIANI, D. A.; GASSMAN, P. W.; FERNANDES, J. G.; GARBOSSA, L. H. P.; SRINIVASAN, R.; BONUMA, N. B.; MENDIONDO, E. M. Review of soil and water assessment tool (SWAT) applications in Brazil: challenges and prospects. International Journal of Agricultural and Biological Engineering, Beijing, Vol. 8, №. 3, 2015.

BRIGHENTI, T. M; BONUMÁ, N. B; CHAFFE, P. L. B. Calibração hierárquica do modelo SWAT em uma bacia hidrográfica Catarinense. Revista Brasileira de Recursos Hídricos. RBRH, vol. 21 no. 1, Porto Alegre jan./mar. 2016.

BONUMÁ, N. B.; REICHERT, J. M.; RODRIGUES, M. F.; MONTEIRO, J. A. F.; ARNOLD, J. G.; SRINIVASAN, R.Modeling surface hydrology, soil erosion, nutrient transport, and future scenarios with the ecohydrological SWAT model in brazilian watersheds and river basins. Tópicos Ci. Solo, v. 9, p. 241-290, 2015.

BUENO, E. de O; OLIVEIRA, V. A. de; VIOLA, M. R; MELLO, C. R. de. Desempenho do Modelo SWAT para Diferentes Critérios de Geração de Unidades de Resposta Hidrológica. Revista Scientia Agrária. vol. 18 n 2 Curitiba Abr/Jun. 2017.

CARVALHO, T. M. Técnicas de medição de vazão por meios convencionais e não convencionais. Revista Brasileira de Geografia Física, Recife, v. 1, n. 1, p. 73-85, maio/ago. 2008.

COOREIA, E. F. G; RIBEIRO, G. P; BAPTISTA, A. C. Modelagem Hidrológica da Bacia Hidrográfica do Rio Bengalas, Nova Friburgo, RJ, Utilizando o Potencial de Geotecnologias na Definição de Áreas de Risco à Inundação. Revista Brasileira de Cartografia, Rio de Janeiro, N. 067/6, p. 1183-1202, Nov/Dez/2015.

DERAEDT, D; COLINET, G; CLAESSENS, H; DEGRÉ, A. Représentation Du couvert forestier dans la modélisation hydrologique :comparaison de deux modèles (synthèse bibliographique), Biotechnol. Agron. Soc. Environ. 2014.

DILE, Y. T.; DAGGUPATI, P.; GEORGE, C.; SRINIVASAN, R.; ARNOLD, J.; Introducing a new open source GIS user interface for the SWAT model. Environmental Modelling \& Software. Vol. 85, November 2016, pages 129-138, INSS 1364-8152, DOI: 10.1016/J. envsoft. 2016.08.004.

EASTON, Z. M.; FUKA, D. R.; WALTER, T.; COWAN, D. M.; SCHNEIDERMAN, E. M.; STEENHUIS, T. S. Re-conceptualizing the soil and water assessment tool (SWAT) model to predict runoff from variable source areas. Journal of Hydrology, 2008.

FAN, F. M.; RAMOS, M. H.; COLLISCHONN, W. Sobre o uso de previsões hidrológicas probabilísticas para tomada de decisão. RBRH vol. 20 no.4, p. 914 - 926.Porto Alegre out./dez. 2015.

FERREIRA, R. S; UAGODA, R. E. S. Análise da predição do balanço hídrico da bacia do ribeirão do Gama-DF através do modelo SWAT. Revista Brasileira de Geografia Física v.10, n.03, 2017.

FERRIGO, S; TÁVORA, B. E; MINOTI, R. T; LIMA, J. E. F. W; KOIDE, S. Avaliação de possíveis impactos das mudanças climáticas e do uso do solo sobre a produção de sedimentos em bacia hidrográfica no Distrito Federal. XI Encontro Nacional de Engenharia de Sedimentos, João Pessoa-PB, Brasil, 01 a 05 de dezembro de 2014. 
FRANCO, A. C. L; OLIVEIRA, D. Y; BONUMÁ, N. B. Transferência Espaço-temporal de Parâmetros no Modelo SWAT. XXII Simpósio Brasileiro de Recursos Hídricos, Florianópolis, SC. 2017.

FENNER, W; MOREIRA, P. S. P; FERREIRA, F. da S; DALLACORT, R; QUEIROZ, T. M; BENTO, T. S. Análise do balanço hídrico mensal para regiões de transição de Cerrado-Floresta e Pantanal, Estado de Mato Grosso. Acta Iguazu, Cascavel, v.3, n.1, p. 72-85, 2014.

GASSMAN, P.W.; SADEGHI, A.M; SRINIVASAN, R. Applications of the SWAT model special section: overciew and insights. Journal of Environmental Quality.2014. Disponívelem:<https://d.sciencesocieties.org/publications/jeq/pdfs/43/1/1>. Acesso em: 07 de agosto de 2017.

INTERNATIONAL ORGANIZATION FOR STANDARDIZATION - ISO. 748 Hydrometry: Measurement of liquid flow in open channels using current-meters or floats. Geneva, 2007.

KUWAJIMA, J. I.; ARANTES, D. M.; ESTIGONI, M. V.; MAUAD, F. F. Proposta da Aplicação do Modelo SWAT como Ferramenta Complementar de Gerenciamento de Recursos Hídricos e Estimativa de Assoreamento em Reservatórios. In: XIV World Water Congress.2011.

MALDONADO, L. H; WENDLAND, E. C; PORTO, M. P. Avaliação de Métodos de Baixo Custo para Medição de Vazão em Córregos. Ambiente \& Água, na Interdisciplinary Journal of Applied Science [on-line] 2015. Disponivel em <HTTP://www.redalyc.org/articulo.oa?id=92838238015. Acessado no dia 28/01/2018.

MARINHO FILHO, G. M. M; ANDRADE, R. S; ZUKOWSKI JUNIOR, J. C; MAGALHÃES FILHO, L. N. L. Modelos Hidrológicos: conceitos e aplicabilidades. Revista de Ciências Ambientais, Canoas, v.6, n.2, p. 35 a 47, 2012.

METCALFE, P; BEVEN, K; FREER, J. Dynamic TOPMODEL: A new implementation in R and its sensitivity to time and space steps. Environmental Modelling \& Software. 2015.

MORIASI D. N, ARNOLD J. G, VAN LIEW M. W, BINGNER R. L, HARMEL R. D, VEITH T. L. Model evaluation guidelines for systematic quantification of accuracy in watershed simulations. Trans ASABE. 2007.

NASH, J. E; J. V. SUTCLIFFE. River Flow Forecasting Through Conceptual Models Part I - A discussion of Principles. Journal of Hydrology. United States, v. 10, n. 3-4, p. 282-290. 1970

NEITSCH, S.L.; ARNOLD J.G.; KINIRY J.R.; WILLIAMS J.R. Soil and Water Assessment Tool Theoretical Documentation Version 2009: TR-406. Texas Water Resources Institute, College Station, TX. 2011.

NIU, S. Q; KNOUFT, J. H. Hydrologic characteristics, food resource abundance, and spatial variation in stream assemblages. Ecohydrology, V. 10, 2017.

PERAZZOLI, M.; PINHEIRO, A.; KAUFMANN, V. Efeitos de cenários de uso do solo sobre o regime hídrico e produção de sedimentos na bacia do Ribeirão Concórdia - SC. Revista Árvore, v. 37, n. 5, 2013.

PEREIRA, A. R. Simplificando o balanço hídrico de Thornthwaite - Mather. Bragantia, Campinas, v. 64, n. 2, p. 311-313, 2005.

PEREIRA, D. R; ULIANA, E. M; MARTINEZ, M. A; SILVA, D. D. Desempenho de um Modelo Hidrológico Concentrado e de um Semi distribuído na Predição de Vazões Diárias. Irriga, Botucatu, v. 21, n. 2, maio - junho, 2016.

PONTES, P. R. M; COLLISCHONN, W; FAN, F. M.; PAIVA, R. C. D.; BUARQUE, D. Modelagem hidrológica e hidráulica de grande escala com propagação inercial de vazões. RBRH vol. 20 no. 4 Porto Alegre out./dez. 2015 p. 888 - 904. 
SILVA, N. D. M; CHRISTOFARO, C. Calibração do modelo hidrológico SWAT para estimativa da vazão em sub-bacia hidrográfica do Rio Jequitinhonha. Revista Geografias, 2017.

SOUZA, P. J. O. P.; FARIAS, V. D. S.; LIMA, M. J. A.; RAMOS, T. F.; SOUSA, A.M.L. Produção de Área Foliar e Biomassa e Produtividade do Feijão-caupi sob Regimes Hídricos em Castanhal, Pará. Revista Caatinga, v. 30, n. 3, p. 748-759, 2017.

TENÓRIO, A. R. M. et al. Mapeamento dos solos da Estação de Pscicultura de Castanhal. 1. ed. Belém: FCAP, 1999. 50 p. (Informe Técnico, 25).

VAN LIEW, M. W.; VEITH, T. L.; BOSCH, D. D.; ARNOLD, J. G. Suitability of SWAT for the conservation effects assessment project: a comparison on USDA-ARS watersheds. Journal of Hydrological Engineering, v. 12, n. 2, 2007.

WANG, X.; SHANG, S.; YANG, W.; CLARY, C. R. D. Simulation of land use-soil interactive effects on water and sediment yields at watershed scale. Ecological Engineering, v. 36, n. 3, p. 328-344, 2010.

Joyse T. Souza dos Santos

Universidade Federal do Pará ;

Programa de Pós-Graduação em Ciências Ambientais, Belém-PA, Brasil.

e-mail: joysetaty@hotmail.com

Contribuição do autor:

Pesquisa realizada como parte de estudo de projeto de tese do autor.

Hildo Giuseppe G. C. Nunes Universidade Federal Rural da Amazônia Programa de Pós-Graduação em Agronomia, Belém-PA, Brasil. e-mail: garibalde13@gmail.com.

Contribuição do autor:

Participação nas coletas de campo, na análise dos dados e escrita do artigo.

Ana Karla dos S. Pontes Universidade Federal de Santa Catarina Programa de Pós-Graduação em Engenharia Ambiental, SC, Brasil. e-mail: anakarlapontes@hotmail.com

Contribuição do autor:

Participação nas coletas de campo, na análise dos dados do artigo.

Adriano M. Leăo de Sousa Universidade Federal Rural da Amazônia Colaborador do Programa de Pós-Graduaçáo em Ciências Ambientais da Universidade Federal do Pará - UFPA (Orientador); Instituto Socioambiental e dos Recursos Hídricos (Professor Adjunto); Laboratório de Pesquisas em Modelagem Hidroclimática da Amazônia (Coordenador); Belém-PA, Brasil. e-mail: adriano.souza@ufra.edu.br.

Contribuição do autor:

Orientador do autor 01; participação na coleta de campo; participação na análise dos dados e escrita do artigo. 